\title{
Analysis of Fractal Wave Equations by Local Fractional Fourier Series Method
}

\author{
Yong-Ju Yang, ${ }^{1}$ Dumitru Baleanu, ${ }^{2,3,4}$ and Xiao-Jun Yang ${ }^{5}$ \\ ${ }^{1}$ School of Mathematics and Statistics, Nanyang Normal University, Nanyang 473061, China \\ ${ }^{2}$ Department of Mathematics and Computer Sciences, Faculty of Arts and Sciences, Cankaya University, 06530 Ankara, Turkey \\ ${ }^{3}$ Department of Chemical and Materials Engineering, Faculty of Engineering, King Abdulaziz University, P.O. Box 80204, Jeddah 21589, \\ Saudi Arabia \\ ${ }^{4}$ Institute of Space Sciences, Magurele, 077125 Bucharest, Romania \\ ${ }^{5}$ Department of Mathematics and Mechanics, China University of Mining and Technology, Xuzhou, Jiangsu 221008, China
}

Correspondence should be addressed to Xiao-Jun Yang; dyangxiaojun@163.com

Received 12 May 2013; Accepted 13 June 2013

Academic Editor: H. Srivastava

Copyright (C) 2013 Yong-Ju Yang et al. This is an open access article distributed under the Creative Commons Attribution License, which permits unrestricted use, distribution, and reproduction in any medium, provided the original work is properly cited.

The fractal wave equations with local fractional derivatives are investigated in this paper. The analytical solutions are obtained by using local fractional Fourier series method. The present method is very efficient and accurate to process a class of local fractional differential equations.

\section{Introduction}

Fractional calculus deals with derivative and integrals of arbitrary orders [1]. During the last four decades, fractional calculus has been applied to almost every field of science and engineering [2-6]. In recent years, there has been a great deal of interest in fractional differential equations [7]. As a result, various kinds of analytical methods were developed [8-18]. For example, there are the exp-function method [8], the variational iteration method $[9,10]$, the homotopy perturbation method [11], the homotopy analysis method [12], the heat-balance integral method [13], the fractional variational iteration method $[14,15]$, the fractional difference method [16], the finite element method [17], the fractional Fourier and Laplace transforms [18], and so on.

Recently, local fractional calculus was applied to deal with problems for nondifferentiable functions; see [19-26] and the references therein. There are also analytical methods for solving the local fractional differential equations, which are referred to in [27-34]. The local fractional series method [3234] was applied to process the local fractional wave equation in fractal vibrating [32] and local fractional heat-conduction equation [33].
More recently, the wave equation on the Cantor sets was considered as $[21,28]$

$$
\frac{\partial^{2 \alpha} u(x, t)}{\partial t^{2 \alpha}}=\frac{\partial^{2 \alpha} u(x, t)}{\partial x^{2 \alpha}} .
$$

Local damped wave equation was written in the form [30]

$$
\frac{\partial^{2 \alpha} u(x, t)}{\partial t^{2 \alpha}}-\frac{\partial^{\alpha} u(x, t)}{\partial t^{\alpha}}-\frac{\partial^{2 \alpha} u(x, t)}{\partial x^{2 \alpha}}=f(x, t),
$$

and local fractional dissipative wave equation in fractal strings was [31]

$$
\begin{aligned}
& \frac{\partial^{2 \alpha} u(x, t)}{\partial t^{2 \alpha}}-\frac{\partial^{\alpha} u(x, t)}{\partial t^{\alpha}} \\
& \quad-\frac{\partial^{2 \alpha} u(x, t)}{\partial x^{2 \alpha}}-\frac{\partial^{\alpha} u(x, t)}{\partial x^{\alpha}}=f(x, t) .
\end{aligned}
$$

In this paper, we investigate the application of local fractional series method for solving the following local fractional wave equation:

$$
\frac{\partial^{2 \alpha} u(x, t)}{\partial t^{2 \alpha}}-\frac{\partial^{\alpha} u(x, t)}{\partial t^{\alpha}}-\frac{\partial^{2 \alpha} u(x, t)}{\partial x^{2 \alpha}}=0,
$$


where initial and boundary conditions are presented as

$$
\begin{gathered}
u(0, t)=u(l, t)=\frac{\partial^{\alpha} u(l, 0)}{\partial x^{\alpha}}=0, \\
u(x, 0)=f(x), \\
\frac{\partial^{\alpha} u(x, 0)}{\partial t^{\alpha}}=g(x) .
\end{gathered}
$$

The organization of the paper is as follows. In Section 2, the basic concepts of local fractional calculus and local fractional Fourier series are introduced. In Section 3, we present a local fractional Fourier series solution of wave equation with local fractional derivative. Two examples are shown in Section 4. Finally, Section 5 is devoted to our conclusions.

\section{Mathematical Tools}

In this section, we present some concepts of local fractional continuity, local fractional derivative, and local fractional Fourier series.

Definition 1 (see $[21,28,30-32]$ ). Suppose that there is

$$
\left|f(x)-f\left(x_{0}\right)\right|<\varepsilon^{\alpha},
$$

with $\left|x-x_{0}\right|<\delta$, for $\varepsilon, \delta>0$ and $\varepsilon, \delta \in R$. Then $f(x)$ is called local fractional continuous at $x=x_{0}$, where $\rho^{\alpha}\left|x-x_{0}\right|^{\alpha} \leq$ $\left|f\left(x_{1}\right)-f\left(x_{2}\right)\right| \leq \tau^{\alpha}\left|x-x_{0}\right|^{\alpha}$ with $\rho, \tau>0$.

Suppose that the function $f(x)$ satisfies the above properties of the local fractional continuity. Then the condition (6) for $x \in(a, b)$ is denoted as

$$
f(x) \in C_{\alpha}(a, b)
$$

where $\operatorname{dim}_{H} f(x)=\alpha$.

Definition 2 (see [19-21]). Let $f(x) \in C_{\alpha}(a, b)$. Local fractional derivative of $f(x)$ of order $\alpha$ at $x=x_{0}$ is given by

$$
\begin{aligned}
D_{x}^{(\alpha)} f\left(x_{0}\right) & =f^{(\alpha)}\left(x_{0}\right) \\
& =\left.\frac{d^{\alpha} f(x)}{d x^{\alpha}}\right|_{x=x_{0}}=\lim _{x \rightarrow x_{0}} \frac{\Delta^{\alpha}\left(f(x)-f\left(x_{0}\right)\right)}{\left(x-x_{0}\right)^{\alpha}},
\end{aligned}
$$

where $\Delta^{\alpha}\left(f(x)-f\left(x_{0}\right)\right) \cong \Gamma(1+\alpha) \Delta\left(f(x)-f\left(x_{0}\right)\right)$.

Definition 3 (see $[19,20,32-34])$. Let $f(x) \in C_{\alpha}(-\infty,+\infty)$, and let $f(x)$ be $2 l$-periodic. For $k \in Z$, local fraction Fourier series of $f(x)$ is defined as

$$
\begin{array}{r}
f(x)=\frac{a_{0}}{2}+\sum_{k=1}^{\infty}\left(a_{n} \cos _{\alpha} \frac{\pi^{\alpha}(k x)^{\alpha}}{l^{\alpha}}\right. \\
\left.+b_{n} \sin _{\alpha} \frac{\pi^{\alpha}(k x)^{\alpha}}{l^{\alpha}}\right),
\end{array}
$$

where the local fraction Fourier coefficients are

$$
\begin{aligned}
& a_{n}=\frac{1}{l^{\alpha}} \int_{-l}^{l} f(x) \cos _{\alpha} \frac{\pi^{\alpha}(k x)^{\alpha}}{l^{\alpha}}(d x)^{\alpha}, \\
& b_{n}=\frac{1}{l^{\alpha}} \int_{-l}^{l} f(x) \sin _{\alpha} \frac{\pi^{\alpha}(k x)^{\alpha}}{l^{\alpha}}(d x)^{\alpha},
\end{aligned}
$$

with local fractional integral given by [21, 29-34]

$$
\begin{aligned}
a_{a}^{I_{b}(\alpha)} f(x) & =\frac{1}{\Gamma(1+\alpha)} \int_{a}^{b} f(t)(d t)^{\alpha} \\
& =\frac{1}{\Gamma(1+\alpha)} \lim _{\Delta t \rightarrow 0} \sum_{j=0}^{j=N-1} f\left(t_{j}\right)\left(\Delta t_{j}\right)^{\alpha}
\end{aligned}
$$

where $\Delta t_{j}=t_{j+1}-t_{j}, \Delta t=\max \left\{\Delta t_{1}, \Delta t_{2}, \Delta t_{j}, \ldots\right\}$ and $\left[t_{j}, t_{j+1}\right], j=0, \ldots, N-1, t_{0}=a, t_{N}=b$, is a partition of the interval $[a, b]$.

In view of (10), the weights of the fractional trigonometric functions are expressed as follows:

$$
\begin{aligned}
& a_{n}=\frac{1 /(\Gamma(1+\alpha)) \int_{-l}^{l} f(x) \cos _{\alpha} n^{\alpha}(\pi x / l)^{\alpha}(d x)^{\alpha}}{1 /(\Gamma(1+\alpha)) \int_{-l}^{l} \cos _{\alpha}^{2} n^{\alpha}(\pi x / l)^{\alpha}(d x)^{\alpha}}, \\
& b_{n}=\frac{1 /(\Gamma(1+\alpha)) \int_{-l}^{l} f(x) \sin _{\alpha} n^{\alpha}(\pi x / l)^{\alpha}(d x)^{\alpha}}{1 /(\Gamma(1+\alpha)) \int_{-l}^{l} \sin _{\alpha}^{2} n^{\alpha}(\pi x / l)^{\alpha}(d x)^{\alpha}} .
\end{aligned}
$$

Lemma 4 (see [21]). If $m$ and $h$ are constant coefficients, then local fractional differential equation with constant coefficients

$$
\frac{d^{2 \alpha} y}{d x^{2 \alpha}}+m \frac{d^{\alpha} y}{d x^{\alpha}}+h y=0 \quad\left(m^{2}-4 h<0\right)
$$

has a family of solution

$$
\begin{aligned}
y(x)= & A E_{\alpha}\left(\frac{-m-i^{\alpha} \sqrt{4 h-m^{2}}}{2} x^{\alpha}\right) \\
& +B E_{\alpha}\left(\frac{-m+i^{\alpha} \sqrt{4 h-m^{2}}}{2} x^{\alpha}\right)
\end{aligned}
$$

with two constants $A$ and $B$.

Proof. See [21].

\section{Solution to Wave Equation with Local Fractional Derivative}

If we have the particular solution of (4) in the form

$$
u(x, t)=\phi(x) T(t)
$$

then we get the equations

$$
\begin{gathered}
\phi^{(2 \alpha)}+\lambda^{2 \alpha} \phi=0 ; \\
T^{(2 \alpha)}+T^{(\alpha)}+\lambda^{2 \alpha} T=0 .
\end{gathered}
$$


with the boundary conditions

$$
\phi(0)=\phi^{(\alpha)}(l)=0 .
$$

Equation (4) has the solution

$$
\phi(x)=C_{1} \cos _{\alpha} \lambda^{\alpha} x^{\alpha}+C_{2} \sin _{\alpha} \lambda^{\alpha} x^{\alpha},
$$

where $C_{1}$ and $C_{2}$ are all constant numbers.

According to (19), for $x=0$ and $x=l$ we get

$$
\begin{gathered}
\phi(0)=C_{1}=0, \\
\phi(l)=\left.\phi(x)\right|_{x=l}=C_{2} \sin _{\alpha} \lambda^{\alpha} l^{\alpha}=0 .
\end{gathered}
$$

Obviously $C_{2} \neq 0$, since otherwise $\phi(x) \equiv 0$.

Hence, we arrive at

$$
\lambda_{n}^{\alpha} l^{\alpha}=n^{\alpha} \pi^{\alpha}
$$

where $n$ is an integer.

We notice

$$
\begin{gathered}
\lambda_{n}^{\alpha}=\left(\frac{n \pi}{l}\right)^{\alpha} \quad(n=0,1,2, \ldots), \\
\phi_{n}(x)=\sin _{\alpha} \lambda_{n}^{\alpha} x^{\alpha} \\
=\sin _{\alpha} n^{\alpha}\left(\frac{\pi x}{l}\right)^{\alpha} \quad(n=0,1,2, \ldots) .
\end{gathered}
$$

For $\lambda^{\alpha}=\lambda_{n}^{\alpha}$ and $0<\rho$, following (17) implies that

$$
\begin{aligned}
\sum_{n=1}^{\infty} T_{n}(t)= & \sum_{n=1}^{\infty} E_{\alpha}\left(-\frac{t^{\alpha}}{2}\right) \\
& \times\left(A_{n} \cos _{\alpha} \rho t^{\alpha}+B_{n} \sin _{\alpha} \rho t^{\alpha}\right),
\end{aligned}
$$

where

$$
\rho=\frac{\sqrt{4(n \pi / l)^{2 \alpha}-1}}{2} .
$$

Therefore,

$$
\begin{aligned}
u_{n}(x, t)= & \phi_{n}(x) T_{n}(t) \\
= & A_{n} \cos _{\alpha} \rho t^{\alpha} \sin _{\alpha} n^{\alpha}\left(\frac{\pi x}{l}\right)^{\alpha} E_{\alpha}\left(-\frac{1}{2} t^{\alpha}\right) \\
& +B_{n} \sin _{\alpha} \rho t^{\alpha} \sin _{\alpha} n^{\alpha}\left(\frac{\pi x}{l}\right)^{\alpha} E_{\alpha}\left(-\frac{1}{2} t^{\alpha}\right) .
\end{aligned}
$$

We now suppose a local fractional Fourier series solution of (4):

$$
\begin{aligned}
u(x, t)= & \sum_{n=1}^{\infty} u_{n}(x, t) \\
= & \sum_{n=1}^{\infty} E_{\alpha}\left(-\frac{t^{\alpha}}{2}\right) \\
& \quad \times\left(A_{n} \cos _{\alpha} \rho t^{\alpha}+B_{n} \sin _{\alpha} \rho t^{\alpha}\right) \sin _{\alpha} n^{\alpha}\left(\frac{\pi x}{l}\right)^{\alpha} .
\end{aligned}
$$

Therefore,

$$
\frac{\partial^{\alpha} u(x, t)}{\partial t^{\alpha}}=\sum_{n=1}^{\infty} \frac{\partial^{\alpha} u_{n}(x, t)}{\partial t^{\alpha}}
$$

where

$$
\begin{aligned}
& \frac{\partial u_{n}(x, t)}{\partial t^{\alpha}} \\
& =-\frac{1}{2} E_{\alpha}\left(-\frac{t^{\alpha}}{2}\right)\left(A_{n} \cos _{\alpha} \rho t^{\alpha}+B_{n} \sin _{\alpha} \rho t^{\alpha}\right) \sin _{\alpha} n^{\alpha}\left(\frac{\pi x}{l}\right)^{\alpha} \\
& \quad+\rho E_{\alpha}\left(-\frac{t^{\alpha}}{2}\right)\left(-A_{n} \sin _{\alpha} \rho t^{\alpha}+B_{n} \cos _{\alpha} \rho t^{\alpha}\right) \sin _{\alpha} n^{\alpha}\left(\frac{\pi x}{l}\right)^{\alpha},
\end{aligned}
$$

with $\rho=\sqrt{\left(4(n \pi / l)^{2 \alpha}-1\right) / 2}$.

Submitting (26) to (5), we have

$$
\begin{aligned}
u(x, 0) & =\sum_{n=1}^{\infty} u_{n}(x, 0) \\
& =\sum_{n=1}^{\infty} A_{n} \sin _{\alpha} n^{\alpha}\left(\frac{\pi x}{l}\right)^{\alpha}=f(x), \\
& \frac{\partial^{\alpha} u(x, t)}{\partial t^{\alpha}} \\
& =\sum_{n=1}^{\infty}\left(-\frac{1}{2} A_{n}+\rho B_{n}\right) \sin _{\alpha} n^{\alpha}\left(\frac{\pi x}{l}\right)^{\alpha}=g(x) .
\end{aligned}
$$

So,

$$
\begin{aligned}
& \sum_{n=1}^{\infty} \rho B_{n} \sin _{\alpha} n^{\alpha}\left(\frac{\pi x}{l}\right)^{\alpha} \\
& \quad=g(x)+\sum_{n=1}^{\infty} \frac{1}{2} A_{n} \sin _{\alpha} n^{\alpha}\left(\frac{\pi x}{l}\right)^{\alpha}=g(x)+\frac{1}{2} f(x) .
\end{aligned}
$$

Let

$$
G(x)=g(x)+\frac{1}{2} f(x) .
$$

In view of (30) and (31), we rewrite

$$
\begin{aligned}
& \sum_{n=1}^{\infty} A_{n} \sin _{\alpha} n^{\alpha}\left(\frac{\pi x}{l}\right)^{\alpha}=f(x), \\
& \sum_{n=1}^{\infty} \rho B_{n} \sin _{\alpha} n^{\alpha}\left(\frac{\pi x}{l}\right)^{\alpha}=G(x) .
\end{aligned}
$$


We now find the local fractional Fourier coefficients of $f(x)$ and $G(x)$, respectively,

$$
\begin{array}{r}
A_{n}=\frac{1 /(\Gamma(1+\alpha)) \int_{0}^{l} f(x) \sin _{\alpha} n^{\alpha}(\pi x / l)^{\alpha}(d x)^{\alpha}}{1 /(\Gamma(1+\alpha)) \int_{0}^{l} \sin _{\alpha}^{2} n^{\alpha}(\pi x / l)^{\alpha}(d x)^{\alpha}} \\
(n=0,1,2, \ldots), \\
\rho B_{n}=\frac{1 /(\Gamma(1+\alpha)) \int_{0}^{l} G(x) \sin _{\alpha} n^{\alpha}(\pi x / l)^{\alpha}(d x)^{\alpha}}{1 /(\Gamma(1+\alpha)) \int_{0}^{l} \sin _{\alpha}^{2} n^{\alpha}(\pi x / l)^{\alpha}(d x)^{\alpha}} \\
(n=0,1,2, \ldots) .
\end{array}
$$

Following (34), we have

$$
\frac{1}{\Gamma(1+\alpha)} \int_{0}^{l} \sin _{\alpha}^{2} n^{\alpha}\left(\frac{\pi x}{l}\right)^{\alpha}(d x)^{\alpha}=\frac{l^{\alpha}}{2 \Gamma(1+\alpha)},
$$

such that

$$
\begin{aligned}
& A_{n}=\frac{2 \int_{0}^{l} f(x) \sin _{\alpha} n^{\alpha}(\pi x / l)^{\alpha}(d x)^{\alpha}}{l^{\alpha}}, \\
& B_{n}=\frac{2 \int_{0}^{l} G(x) \sin _{\alpha} n^{\alpha}(\pi x / l)^{\alpha}(d x)^{\alpha}}{\rho l^{\alpha}} .
\end{aligned}
$$

Thus, we get the solution of (4):

$$
u(x, t)=\sum_{n=1}^{\infty} u_{n}(x, t)
$$

where

$$
\begin{aligned}
u_{n}(x, t)= & E_{\alpha}\left(-\frac{t^{\alpha}}{2}\right) \\
& \times\left(A_{n} \cos _{\alpha} \rho t^{\alpha}+B_{n} \sin _{\alpha} \rho t^{\alpha}\right) \sin _{\alpha} n^{\alpha}\left(\frac{\pi x}{l}\right)^{\alpha},
\end{aligned}
$$

with

$$
\begin{aligned}
& A_{n}=\frac{2 \int_{0}^{l} f(x) \sin _{\alpha} n^{\alpha}(\pi x / l)^{\alpha}(d x)^{\alpha}}{l^{\alpha}} \quad(n=0,1,2, \ldots), \\
& B_{n}=\frac{2 \int_{0}^{l} G(x) \sin _{\alpha} n^{\alpha}(\pi x / l)^{\alpha}(d x)^{\alpha}}{\rho^{\alpha}} \quad(n=0,1,2, \ldots),
\end{aligned}
$$

with

$$
G(x)=g(x)+\frac{1}{2} f(x) .
$$

\section{Illustrative Examples}

In order to illustrate the above result in this section, we give two examples.
Let us consider (4) subject to initial and boundary conditions

$$
\begin{gathered}
u(0, t)=u(l, t)=\frac{\partial^{\alpha} u(l, 0)}{\partial x^{\alpha}}=0, \\
u(x, 0)=f(x)=\frac{x^{\alpha}}{\Gamma(1+\alpha)}, \\
\frac{\partial^{\alpha} u(x, 0)}{\partial t^{\alpha}}=g(x)=\frac{x^{\alpha}}{\Gamma(1+\alpha)} .
\end{gathered}
$$

In view of (40), we have

$$
G(x)=g(x)+\frac{1}{2} f(x)=\frac{3}{2} \frac{x^{\alpha}}{\Gamma(1+\alpha)},
$$

such that

$$
\begin{aligned}
A_{n} & =\frac{2 \int_{0}^{l}\left(x^{\alpha} / \Gamma(1+\alpha)\right) \sin _{\alpha} n^{\alpha}(\pi x / l)^{\alpha}(d x)^{\alpha}}{l^{\alpha}} \\
& =\frac{2 \Gamma(1+\alpha)}{l^{\alpha}}{ }_{0} I_{l}^{(\alpha)} \frac{x^{\alpha}}{\Gamma(1+\alpha)} \sin _{\alpha} n^{\alpha}\left(\frac{\pi x}{l}\right)^{\alpha} \\
& =\frac{2 \Gamma(1+\alpha)}{(n \pi)^{\alpha}}\left\{\frac{l^{\alpha}}{\Gamma(1+\alpha)} \cos _{\alpha} n^{\alpha}\left(\frac{\pi x}{l}\right)^{\alpha}\right. \\
B_{n} & \left.\left.=\frac{3 \int_{0}^{l}\left(x^{\alpha} / \Gamma(1+\alpha)\right) \sin _{\alpha} n^{\alpha}(\pi x / l)^{\alpha}(d x)^{\alpha}}{\rho l^{\alpha}}\right)^{\alpha}\left[\cos _{\alpha} n^{\alpha}\left(\frac{\pi x}{l}\right)^{\alpha}-1\right]\right\}, \\
& =\frac{3 \Gamma(1+\alpha)}{\rho l^{\alpha}}{ }_{0} I_{l}^{(\alpha)} \frac{x^{\alpha}}{\Gamma(1+\alpha)} \sin _{\alpha} n^{\alpha}\left(\frac{\pi x}{l}\right)^{\alpha} \\
& =-\frac{3 \Gamma(1+\alpha)}{\rho(n \pi)^{\alpha}}\left[\frac{l^{\alpha}}{\Gamma(1+\alpha)} \cos _{\alpha} n^{\alpha}\left(\frac{\pi x}{l}\right)^{\alpha}\right. \\
& \left.-\left(\frac{l}{n \pi}\right)^{\alpha} \sin _{\alpha} n^{\alpha}\left(\frac{\pi x}{l}\right)^{\alpha}\right] .
\end{aligned}
$$

Hence,

$$
\begin{aligned}
u(x, t)= & \sum_{n=1}^{\infty} E_{\alpha}\left(-\frac{t^{\alpha}}{2}\right) \\
& \times\left(A_{n} \cos _{\alpha} \rho t^{\alpha}+B_{n} \sin _{\alpha} \rho t^{\alpha}\right) \sin _{\alpha} n^{\alpha}\left(\frac{\pi x}{l}\right)^{\alpha},
\end{aligned}
$$

where

$$
A_{n}=-\frac{2 \Gamma(1+\alpha)}{\rho(n \pi)^{\alpha}}\left[\frac{l^{\alpha}}{\Gamma(1+\alpha)} \cos _{\alpha} n^{\alpha}\left(\frac{\pi x}{l}\right)^{\alpha}\right.
$$

$$
\begin{aligned}
&\left.-\left(\frac{l}{n \pi}\right)^{\alpha} \sin _{\alpha} n^{\alpha}\left(\frac{\pi x}{l}\right)^{\alpha}\right], \\
& B_{n}=-\frac{3 \Gamma(1+\alpha)}{\rho(n \pi)^{\alpha}}\left[\frac{l^{\alpha}}{\Gamma(1+\alpha)} \cos _{\alpha} n^{\alpha}\left(\frac{\pi x}{l}\right)^{\alpha}\right. \\
&\left.-\left(\frac{l}{n \pi}\right)^{\alpha} \sin _{\alpha} n^{\alpha}\left(\frac{\pi x}{l}\right)^{\alpha}\right] .
\end{aligned}
$$


In view of (4), our second example is initial and boundary conditions as follows:

$$
\begin{gathered}
u(0, t)=u(l, t)=\frac{\partial^{\alpha} u(l, 0)}{\partial x^{\alpha}}=0, \\
u(x, 0)=f(x)=\frac{x^{\alpha}}{\Gamma(1+\alpha)}, \\
\frac{\partial^{\alpha} u(x, 0)}{\partial t^{\alpha}}=g(x)=0 .
\end{gathered}
$$

Following (40), we get

$$
G(x)=\frac{1}{2} \frac{x^{\alpha}}{\Gamma(1+\alpha)} .
$$

Hence, we obtain

$$
\begin{aligned}
A_{n} & =\frac{2 \int_{0}^{l}\left(x^{\alpha} / \Gamma(1+\alpha)\right) \sin _{\alpha} n^{\alpha}(\pi x / l)^{\alpha}(d x)^{\alpha}}{l^{\alpha}} \\
& =\frac{2 \Gamma(1+\alpha)}{l^{\alpha}}{ }_{0} I_{l}^{(\alpha)} \frac{x^{\alpha}}{\Gamma(1+\alpha)} \sin _{\alpha} n^{\alpha}\left(\frac{\pi x}{l}\right)^{\alpha} \\
& =-\frac{2 \Gamma(1+\alpha)}{\rho(n \pi)^{\alpha}}\left[\frac{l^{\alpha}}{\Gamma(1+\alpha)} \cos _{\alpha} n^{\alpha}\left(\frac{\pi x}{l}\right)^{\alpha}\right. \\
B_{n} & \left.=\frac{\int_{0}^{l}\left(x^{\alpha} / \Gamma(1+\alpha)\right) \sin _{\alpha} n^{\alpha}(\pi x / l)^{\alpha}(d x)^{\alpha}}{\rho l^{\alpha}} \sin _{\alpha} n^{\alpha}\left(\frac{\pi x}{l}\right)^{\alpha}\right], \\
& =\frac{\Gamma(1+\alpha)}{\rho l^{\alpha}}{ }_{0} I_{l}^{(\alpha)} \frac{x^{\alpha}}{\Gamma(1+\alpha)} \sin _{\alpha} n^{\alpha}\left(\frac{\pi x}{l}\right)^{\alpha} \\
& =-\frac{\Gamma(1+\alpha)}{\rho(n \pi)^{\alpha}}\left[\frac{l^{\alpha}}{\Gamma(1+\alpha)} \cos _{\alpha} n^{\alpha}\left(\frac{\pi x}{l}\right)^{\alpha}\right. \\
& \left.-\left(\frac{l}{n \pi}\right)^{\alpha} \sin _{\alpha} n^{\alpha}\left(\frac{\pi x}{l}\right)^{\alpha}\right] .
\end{aligned}
$$

So,

$$
\begin{aligned}
u(x, t)= & \sum_{n=1}^{\infty} E_{\alpha}\left(-\frac{t^{\alpha}}{2}\right) \\
& \times\left(A_{n} \cos _{\alpha} \rho t^{\alpha}+B_{n} \sin _{\alpha} \rho t^{\alpha}\right) \sin _{\alpha} n^{\alpha}\left(\frac{\pi x}{l}\right)^{\alpha},
\end{aligned}
$$

with

$$
\begin{gathered}
A_{n}=\frac{2 \Gamma(1+\alpha)}{(n \pi)^{\alpha}}\left\{\frac{l^{\alpha}}{\Gamma(1+\alpha)} \sin _{\alpha} n^{\alpha}\left(\frac{\pi x}{l}\right)^{\alpha}\right. \\
\left.-\left(\frac{l}{n \pi}\right)^{\alpha}\left[\cos _{\alpha} n^{\alpha}\left(\frac{\pi x}{l}\right)^{\alpha}-1\right]\right\}, \\
B_{n}=-\frac{\Gamma(1+\alpha)}{\rho(n \pi)^{\alpha}}\left[\frac{l^{\alpha}}{\Gamma(1+\alpha)} \cos _{\alpha} n^{\alpha}\left(\frac{\pi x}{l}\right)^{\alpha}\right. \\
\left.-\left(\frac{l}{n \pi}\right)^{\alpha} \sin _{\alpha} n^{\alpha}\left(\frac{\pi x}{l}\right)^{\alpha}\right] .
\end{gathered}
$$

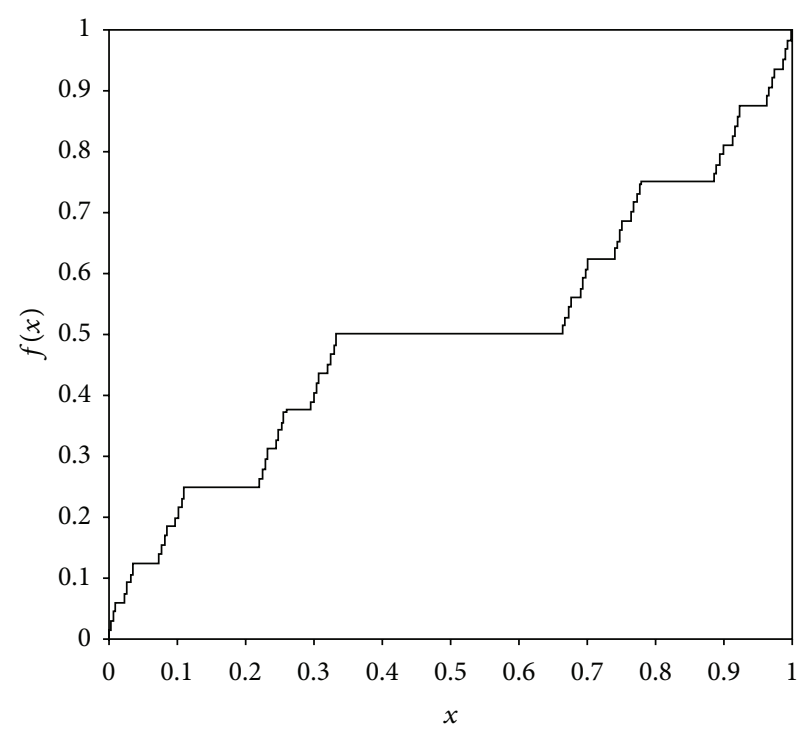

Figure 1: For $\alpha=\ln 2 / \ln 3$, graph of a Lebesgue-Cantor staircase function shown at $x \in[0,1]$.

We notice that fraction boundary condition is expressed as a Lebesgue-Cantor staircase function $[21,32]$; namely,

$$
\begin{aligned}
u(x, 0) & =f(x)=H_{\alpha}(C \cap(0, x)) \\
& ={ }_{0} I_{x}{ }^{(\alpha)} 1=\frac{x^{\alpha}}{\Gamma(1+\alpha)},
\end{aligned}
$$

where $C$ is any fractal set and the fractal dimension of $x^{\alpha} / \Gamma(1+\alpha)$ is $\alpha$. For $x \in[0,1]$ the graph of the LebesgueCantor staircase function (52) is shown in Figure 1 when fractal dimension is $\alpha=\ln 2 / \ln 3$.

\section{Conclusions}

The present work expresses the local fractional Fourier series solution to wave equations with local fractional derivative. Two examples are given to illustrat approximate solutions for wave equations with local fractional derivative resulting from local fractional Fourier series method. The results obtained from the local fractional analysis seem to be general since the obtained solutions go back to the classical one when fractal dimension $\alpha=1$; namely, it is a process from fractal geometry to Euclidean geometry. Local fractional Fourier series method is one of very efficient and powerful techniques for finding the solutions of the local fractional differential equations. It is also worth noting that the advantage of the local fractional differential equations displays the nondifferential solutions, which show the fractal and local behaviors of moments. However, the classical Fourier series is used to handle the continuous functions.

\section{References}

[1] A. A. Kilbas, H. M. Srivastava, and J. J. Trujillo, Theory and Applications of Fractional Differential Equations, vol. 204 
of North-Holland Mathematics Studies, Elsevier Science B.V., Amsterdam, The Netherlands, 2006.

[2] F. Mainardi, Fractional Calculus and Waves in Linear Viscoelasticity, Imperial College Press, London, UK, 2010.

[3] J. Klafter, S. C. Lim, and R. Metzler, Fractional Dynamics in Physics: Recent Advances, World Scientific, Singapore, 2012.

[4] G. M. Zaslavsky, Hamiltonian Chaos and Fractional Dynamics, Oxford University Press, Oxford, UK, 2008.

[5] D. Baleanu, J. A. Tenreiro Machado, and A. C. J. Luo, Fractional Dynamics and Control, Springer, New York, NY, USA, 2012.

[6] J. A. Tenreiro Machado, A. C. J. Luo, and D. Baleanu, Nonlinear Dynamics of Complex Systems: Applications in Physical, Biological and Financial Systems, Springer, New York, NY, USA, 2011.

[7] I. Podlubny, Fractional Differential Equations, vol. 198 of Mathematics in Science and Engineering, Academic Press, New York, NY, USA, 1999.

[8] S. A. El-Wakil, M. A. Madkour, and M. A. Abdou, "Application of Exp-function method for nonlinear evolution equations with variable coefficients," Physics Letters A, vol. 369, no. 1-2, pp. 6269, 2007.

[9] S. Das, "Analytical solution of a fractional diffusion equation by variational iteration method," Computers \& Mathematics with Applications, vol. 57, no. 3, pp. 483-487, 2009.

[10] S. Momani and Z. Odibat, "Comparison between the homotopy perturbation method and the variational iteration method for linear fractional partial differential equations," Computers \& Mathematics with Applications, vol. 54, no. 7-8, pp. 910-919, 2007.

[11] S. T. Mohyud-Din, M. A. Noor, and K. I. Noor, "Some relatively new techniques for nonlinear problems," Mathematical Problems in Engineering, vol. 2009, Article ID 234849, 25 pages, 2009.

[12] H. Jafari and S. Seifi, "Homotopy analysis method for solving linear and nonlinear fractional diffusion-wave equation," Communications in Nonlinear Science and Numerical Simulation, vol. 14, no. 5, pp. 2006-2012, 2009.

[13] J. Hristov, "Heat-balance integral to fractional (half-time) heat diffusion sub-model," Thermal Science, vol. 14, no. 2, pp. 291-316, 2010.

[14] G.-c. Wu and E. W. M. Lee, "Fractional variational iteration method and its application," Physics Letters A, vol. 374, no. 25, pp. 2506-2509, 2010.

[15] Y. Khan, N. Faraz, A. Yildirim, and Q. Wu, "Fractional variational iteration method for fractional initial-boundary value problems arising in the application of nonlinear science," Computers \& Mathematics with Applications, vol. 62, no. 5, pp. 2273-2278, 2011.

[16] Z. Zhao and C. Li, "Fractional difference/finite element approximations for the time-space fractional telegraph equation," Applied Mathematics and Computation, vol. 219, no. 6, pp. 29752988, 2012.

[17] W. Deng, "Finite element method for the space and time fractional Fokker-Planck equation," SIAM Journal on Numerical Analysis, vol. 47, no. 1, pp. 204-226, 2008.

[18] D. Baleanu, K. Diethelm, E. Scalas, and J. J. Trujillo, Fractional Calculus Models and Numerical Methods, vol. 3 of Series on Complexity, Nonlinearity and Chaos, World Scientific, Hackensack, NJ, USA, 2012.

[19] X. J. Yang, Local Fractional Functional Analysis and Its Applications, Asian Academic, Hong Kong, China, 2011.

[20] X. J. Yang, "Local fractional integral transforms," Progress in Nonlinear Science, vol. 4, pp. 1-225, 2011.
[21] X. J. Yang, Advanced Local Fractional Calculus and Its Applications, World Science, New York, NY, USA, 2012.

[22] K. M. Kolwankar and A. D. Gangal, "Local fractional FokkerPlanck equation," Physical Review Letters, vol. 80, no. 2, pp. 214217, 1998.

[23] A. Carpinteri and A. Sapora, "Diffusion problems in fractal media defined on Cantor sets," ZAMM Zeitschrift fur Angewandte Mathematik und Mechanik, vol. 90, no. 3, pp. 203-210, 2010.

[24] G. Jumarie, "Probability calculus of fractional order and fractional Taylor's series application to Fokker-Planck equation and information of non-random functions," Chaos, Solitons and Fractals, vol. 40, no. 3, pp. 1428-1448, 2009.

[25] Y. Khan, Q. Wu, N. Faraz, A. Yildirim, and M. Madani, "A new fractional analytical approach via a modified RiemannLiouville derivative," Applied Mathematics Letters, vol. 25, no. 10, pp. 1340-1346, 2012.

[26] Y. Khan, N. Faraz, S. Kumar, and A. Yildirim, "A coupling method of homotopy perturbation and Laplace transformation for fractional models," "Politehnica" University of Bucharest, vol. 74, no. 1, pp. 57-68, 2012.

[27] M. S. Hu, D. Baleanu, and X. J. Yang, "One-phase problems for discontinuous heat transfer in fractal media," Mathematical Problems in Engineering, vol. 2013, Article ID 358473, 3 pages, 2013.

[28] W.-H. Su, X.-J. Yang, H. Jafari, and D. Baleanu, "Fractional complex transform method for wave equations on Cantor sets within local fractional differential operator," Advances in Difference Equations, vol. 2013, article 97, 2013.

[29] X. J. Yang and D. Baleanu, "Fractal heat conduction problem solved by local fractional variation iteration method," Thermal Science, vol. 17, no. 2, pp. 625-628, 2013.

[30] W.-H. Su, D. Baleanu, X.-J. Yang, and H. Jafari, "Damped wave equation and dissipative wave equation in fractal strings within the local fractional variational iteration method," Fixed Point Theory and Applications, vol. 2013, article 89, 2013.

[31] Y. J. Yang, D. Baleanu, and X. J. Yang, "A local fractional variational iteration method for Laplace equation within local fractional operators," Abstract and Applied Analysis, vol. 2013, Article ID 202650, 6 pages, 2013.

[32] M.-S. Hu, R. P. Agarwal, and X.-J. Yang, "Local fractional Fourier series with application to wave equation in fractal vibrating string," Abstract and Applied Analysis, vol. 2012, Article ID 567401, 15 pages, 2012.

[33] Y. Zhang, A. Yang, and X. J. Yang, "1-D heat conduction in a fractal medium: a solution by the local fractional Fourier series method," Thermal Science, 2013.

[34] G. A. Anastassiou and O. Duman, In Applied Mathematics and Approximation Theory, Springer, New York, NY, USA, 2013. 


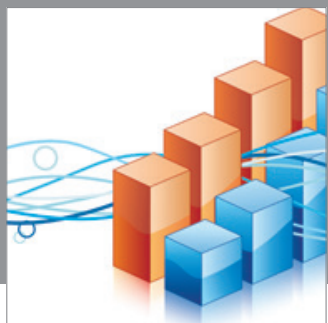

Advances in

Operations Research

mansans

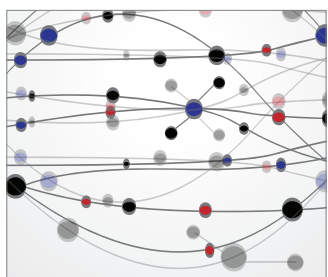

The Scientific World Journal
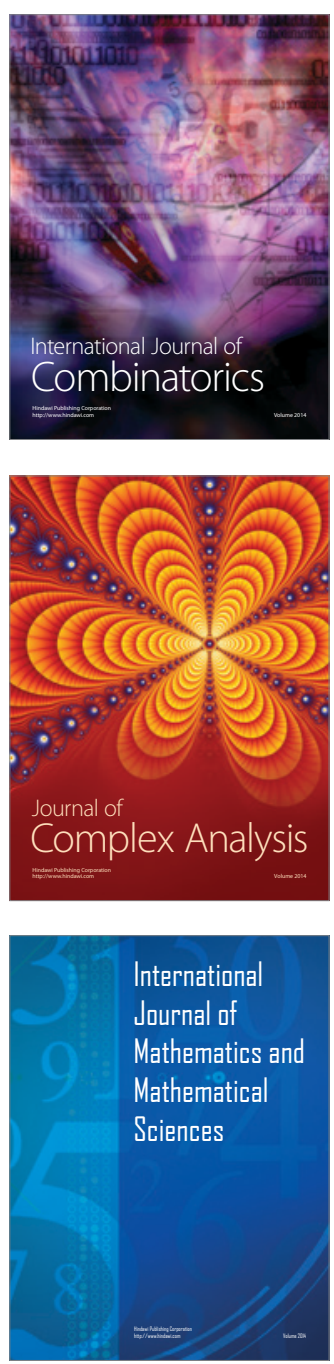
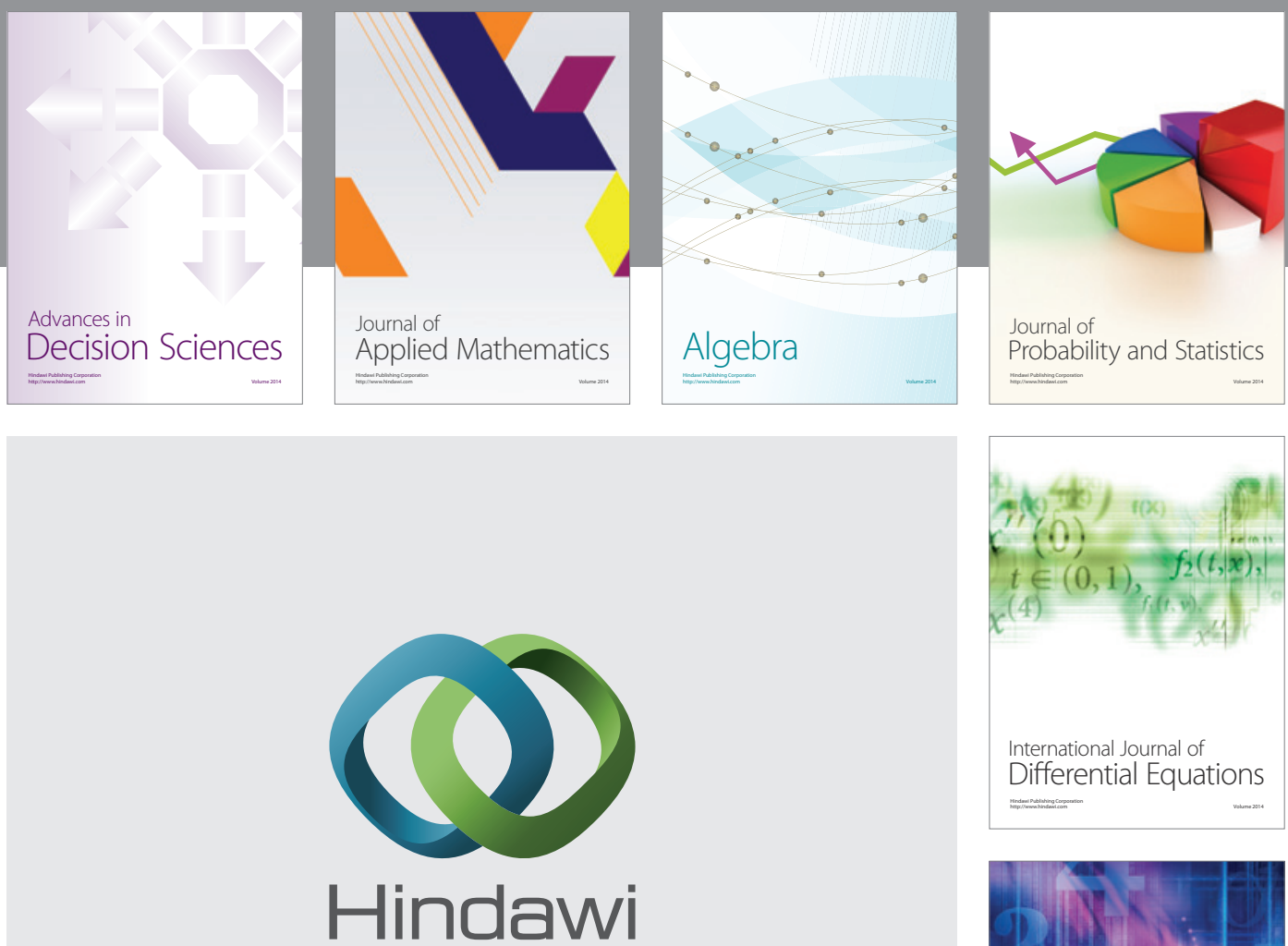

Submit your manuscripts at http://www.hindawi.com
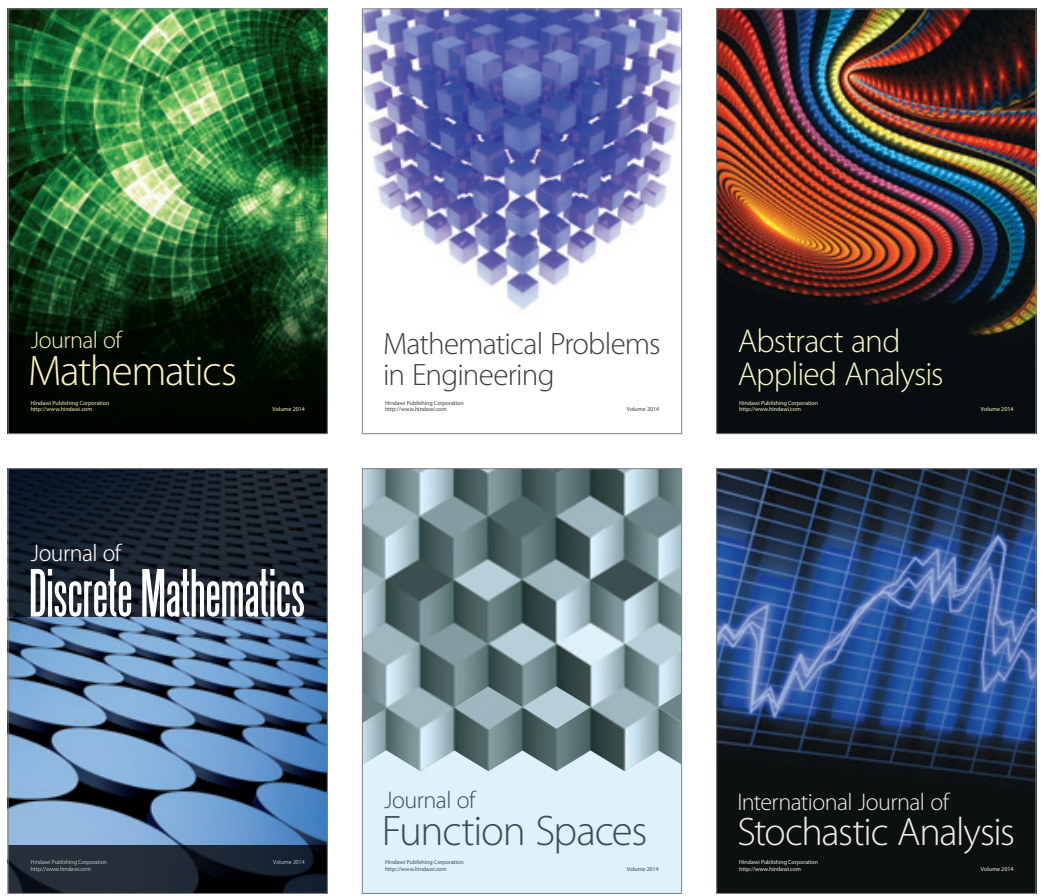

Journal of

Function Spaces

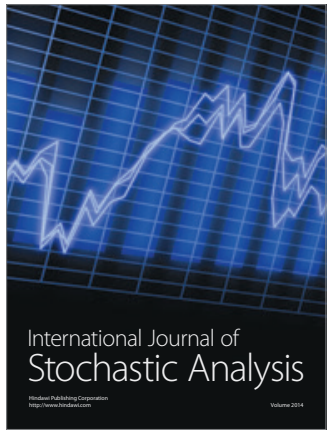

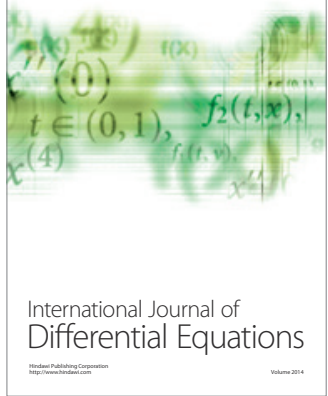
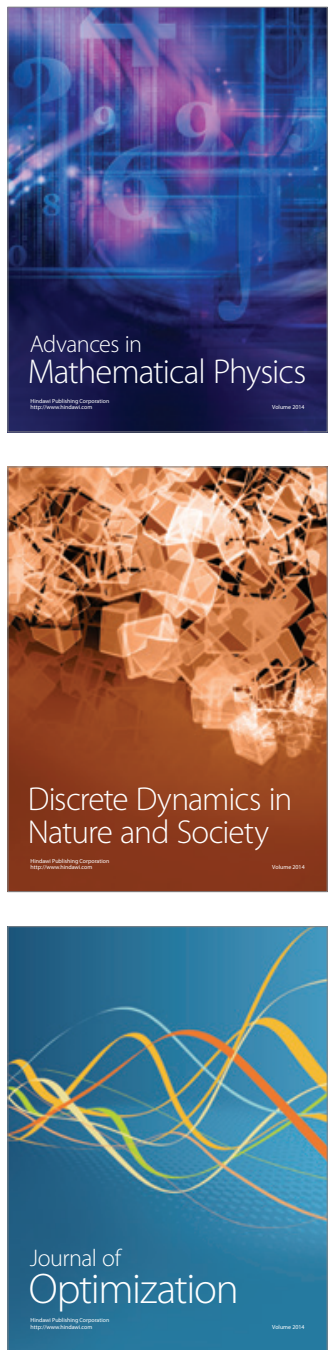